\title{
Téoros
}

Revue de recherche en tourisme

\section{Les Américains au Québec. Le géant endormi ?}

\section{Jean Stafford et Bruno Sarrasin}

Volume 16, numéro 2, été 1997

URI : https://id.erudit.org/iderudit/1074585ar

DOI : https://doi.org/10.7202/1074585ar

Aller au sommaire du numéro

Éditeur(s)

Université du Québec à Montréal

ISSN

0712-8657 (imprimé)

1923-2705 (numérique)

Découvrir la revue

Citer cette note

Stafford, J. \& Sarrasin, B. (1997). Les Américains au Québec. Le géant endormi ? Téoros, 16(2), 53-55. https://doi.org/10.7202/1074585ar d'utilisation que vous pouvez consulter en ligne.

https://apropos.erudit.org/fr/usagers/politique-dutilisation/ 


\title{
Les Américains au Québec. LE GÉANT ENDORMI?
}

\author{
Jean Stafford, professeur \\ Département d'études urbaines et touristiques - UQAM \\ Bruno Sarrasin, chargé de cours \\ Département d'études urbaines et touristiques - UQAM
}

Cette nowvelle chronique hors thème porte sur la conjoncture touristique. Elle propose un portrait simplifié de la situation da tourisme à partir d'une clientele précise et des donnés disponibles. Les notions usuelles de l'analyse prévisionnelle seront utilisées : la tendance (passée, présente et future), la saisonnalité, le cycle et les mouvements irréguliers. Des prévisions seront faites pour les cinq prochaines années.

Les Américains constituent le marché international le plus important pour le Québec en terme de visiteurs!. En 1995, ces visites représentaient $75 \%$ du tourisme international, avec un volume équivalent à la clientèle des Canadiens des autres provinces qui voyagent au Québec (Tourisme Québec, $1997: 45$ ). On évoque souvent le marché américain comme un bassin potentiel de plusieurs millions de visiteurs, prêts à traverser la frontière à la première invitation. Pourtant, l'évolution de ce marché sur une période de 20 ans montre peu de changements importants et témoigne de ce que l'on pourrait attendre de ce marché dans l'avenir.

\section{TENDANCES}

De 1977 à 1981 le taux d'accroissement annuel moyen ạ été de $1,4 \%$ (tableau 1 ). Il a légèrement augmenté dans les années suivantes (1982-1986) pour atteindre près de $2,7 \%$. La période 1987-1991 fut par ailleurs désastreuse avec une baisse annuelle de $-3,24 \%$, malgré un taux de change favorable aux Américains. Il faut se rappeler que ces années ont vu la croissance annuelle de l'ếconomie américaine passer de $3,8 \%$ en 1988 à $-0,7 \%$ en 1991, avec un effet négatif sur les revenus et, par extension, sur les vacances. La dernière période (1992-1996) a connu une légère amélioration avec une hausse de $2 \%$ des arrivées de touristes américains au Québec, suivant ainsi le cours de la croissance économique observée en Amérique du Nord depuis les cinq dernières annếes.

Tableau 1 :

Laarrivée des touristes américains au Québec ; les taux d'accroissement annuels moyens (moyenne géométrique), de 1977 à 1996 par périodes de cinq années.

\begin{tabular}{|c|c|}
\hline $\begin{array}{l}\text { Laarrivée des touristes am } \\
\text { (moyenne géom }\end{array}$ & $\begin{array}{l}\text { aux d'accroissement annuels moyens } \\
\text { ar périodes de cinq années. }\end{array}$ \\
\hline Années & Taux d"acroissement en $\%$ \\
\hline $1977-1981$ & 1,39 \\
\hline 1982-1986 & 2,67 \\
\hline $1987-1991$ & $-3,24$ \\
\hline $1992-1996$ & 2,01 \\
\hline $1977-1996$ & 0,01 \\
\hline
\end{tabular}

Source: nos calculs, a partir des donnes de Statistique Canada.
L'analyse de l'ensemble des périodes entre 1977 et 1996 indique un taux de croissance nul $(0,01 \%)$. $\mathrm{A}$ toutes fins utiles on peut dire que cette clientèle touristique est stationnaire $\$$ et il ne faut pas s'attendre à des changements importants, à ce titre, dans les cinq prochaines années.

\section{LA SAISONNALITÉ}

La saisonnalité des clientèles américaines montre une très grande stabilité. Sur une vingtaine d'années les variations sont relativement faibles. Dans les dix premières années (1977-1981 et 1982-1986) les seuls changements intéressants apparaissent en mars où le coefficient saisonnier passe de 75 à 67 (tableau 2). Pour les mois d'avril à décembre les variations sont faibles ou nulles. Pour les périodes 1987-1991 et 1992-1996 nous pouvons remarquer une baisse de $-8 \%$ en juillet et août par rapport aux dix années précédentes, au profit d'un plus fort étalement 
Tableau 2:

Les arrivées des touristes américains au Québec ; I'évolution des coefficients saisonniers" de 1977 à 1996 par périodes de cinq années.

\begin{tabular}{|lccccccccccccc|}
\hline Années & \multicolumn{10}{c|}{ Mois } \\
\hline & 01 & 02 & 03 & 04 & 05 & 06 & 07 & 08 & 09 & 10 & 11 & 12 \\
\hline $1977-1981$ & 58 & 70 & 75 & 84 & 101 & 115 & 174 & 168 & 116 & 96 & 72 & 71 \\
\hline $1982-1986$ & 57 & 66 & 67 & 81 & 101 & 118 & 175 & 171 & 117 & 101 & 73 & 71 \\
\hline $1987-1991$ & 62 & 69 & 72 & 80 & 102 & 125 & 167 & 163 & 117 & 97 & 72 & 75 \\
\hline $1992-1996$ & 57 & 69 & 71 & 83 & 103 & 123 & 167 & 162 & 118 & 102 & 71 & 75 \\
\hline Moyennes & 58 & 68 & 71 & 82 & 102 & 120 & 171 & 166 & 117 & 99 & 72 & 73 \\
\hline
\end{tabular}

1 Ces coefficients sont calcules à partir de la máthode de cócomposition des séries chronologiques :

a I'side du logiciel SPSS, en utilisent les donneses de statistique Canada.

\section{Tableau 3:}

Les arrivées des touristes américains au Québec, de 1977 à 1996 ; synthèse des éléments de décomposition de la série chronologique.

\begin{tabular}{|lll|}
\hline Tendance & $=$ & Faible $; 1 \cdot 2 \%$ \\
\hline Saisonnalité & $=$ & Très stable, peu de variation \\
\hline Cycle & $=$ & Très peu accentué $; 4,2 \mathrm{a} 5,3$ \\
\hline Mouvements irréguliers & $=$ & Assez faible $; \pm 10 \%$ \\
\hline
\end{tabular}

Tableau 4 :

Visites-province des Américains au Québec en 1990 et 1995, avec taux d'accroissement annuel moyen (moyenne géométrique).

\begin{tabular}{|c|c|c|c|c|c|}
\hline \multirow{3}{*}{ Régions } & \multicolumn{4}{|c|}{ Visites-province (000) } & \multirow{3}{*}{$\begin{array}{c}\text { TAAM } \\
\%\end{array}$} \\
\hline & \multicolumn{2}{|c|}{1990} & \multicolumn{2}{|c|}{1995} & \\
\hline & Nbre & $\%$ & Nbre & $\%$ & \\
\hline Nouvelle-Angleterre & 651 & 35 & 669 & 34 & 0,7 \\
\hline Atlantique Centre & 581 & 31 & 541 & 28 & $-1,8$ \\
\hline Atlantique Sud & 219 & 12 & 237 & 12 & 2 \\
\hline Centre Nord-Est & 182 & 10 & 222 & 11 & 5,1 \\
\hline Centre Sud-Duest & 42 & 2 & 45 & 2 & 1,7 \\
\hline Pacifique & 98 & 5 & 128 & 7 & 6,9 \\
\hline Autres régions & 91 & 5 & 103 & 4 & 3,1 \\
\hline TOTAL & 1864 & 100 & 1945 & 100 & 1,1 \\
\hline
\end{tabular}

Sourca: Tourisme Québec (1997; 51 ). des arrivées en juin et, à un degré moindre, en décembre. Les dates des grands événements d'été à Montréal auraientelles contribué à modifier la saisonnalité ? Il semble qu'avec la notoriété grandissante des Grand Prix du Canada, Festival de Jazz, Festival Juste pour rire, Mondial de la bière, etç, les Américains en visite au Québec aient pu tenir compte de ces événements pour planifier leurs vacances. En effet, en 1994 près de $30 \%$ d'entre eux avaient participé à des festivals et foires, à des événements culturels ou à des évếnements sportifs (Tourisme Québec, 1995 : 33). Dans l'ensemble, les arrivées restent principalement concentrées en juillet-août et, dans une moindre mesure, en juin et septembre, la saisonnalité représentant une certaine forme d'invariance, gage de stabilité de la clientèle américaine.

\section{UN MARCHÉ INERTE OU EN POSSIBLE DÉVELOPPEMENT ?}

La caractéristique qui décrit le mieux le marché américain reste sans doute la constance, pour ne pas dire la stagnation. Le tableau 3 résume bien cette situation, en montrant que pour l'ensemble du marché très peu de changements se sont produits depuis 20 ans, sur le plan de la tendance $(+1-2 \%)$, de la saisonnalité (stable), du cycle (faible) et du mouvement irrégulier $( \pm 10 \%)$. Le marché américain serait-il atrophié pour le Québec, essoufflé par les aléas de l'économie ou carrément saturé ? Le Québec, comme destination touristique, a-t-il du mal a tailler sa juste part du marche américain dans un contexte de farouche compétitivité que l'on a maintenant l'habitude d'associer à la mondialisation ?

Une chose semble de plus en plus claire, c'est que les régions utilisćes pour segmenter ce marché (tableau 4) ont une évolution souvent différente et fonctionnent en quelque sorte à partir du principe des vases communiquants : lorsqu'un vase se remplit l'autre se vide, quand on observe une croissance annuelle moyenne pour les régions du Pacifique ou du Centre Nord-Est, de 1990 à 1995, on assiste à une baisse ou à une stagnation des segments qui représentent le bassin de clientèle primaire, tels la Nouvelle-Angleterre ou l'Atlantique Centre. En d'autres termes, au moment 
où de nouvelles clientèles américaines éloignées \& découvrent * le Québec comme destination touristique, les clientèles traditionnelles semblent chercher d'autres avenues de vacances, dans un environnement qui ressemble, sur le plan géographique, peut-être un peu moins au leur.

Sur le plan touristique, le Québec représente en quelque sorte « l'arrièrecour $\$$ du géant américain, les visiteurs américains faisant chez nous un court séjour avant de repartir aussitôt (Stafford et Blier, $1996: 19$ ). A l'échelle mondiale, on observe que les flux touristiques vont généralement dans le même sens que les échanges économiques. Même si les Américains visitent le Québec surtout par agrément $(57,3 \%), 18,1 \%$ d'entre eux viennent pour affaires ou pour un congrès et représentent une forme de clientèle * captive s des relations commerciales entre le Québec et les États-Unis. Cette clientèle d'affaires provient surtout des régions limitrophes comme la NouvelleAngleterre et l'Atlantique Centre $62,2 \%$ des visiteurs américains en 1995 - en particulier des états du New Hampshire, du Vermont et du Maine. Si une stagnation ou une baisse est à prévoir pour les visiteurs d'agrément en provenance de ces régions, on peut s'attendre à une légère croissance de la clientèle d'affaires dans le moyen terme.

La paralysie actuelle du marché américain au Québec n'est peut-être pas définitive, mais l'idée voulant qu'il représenté la principale voie de l'avenir a de bonnes raisons d'être dépassée, pour ne pas dire trépassée. En posant l'hypothèse d'un cycle conjoncturel positif jusqu'en 1999 et légèrement négatif en 2000 et en 2001 , on peut s'attendre à une croissance de $5 \%$ pour l'ensemble du marché en 1997 , suivie d'une baisse régulière moyenne d'environ $4 \%$ par année jusqu'en 2001 (graphique 1). Certaines clientèles peuvent cependant aller à contresens de la tendance généralement observée. La clientèle d'affaires, les retraités - de plus en plus jeunes et mieux nantis - qui utilisent l'avion plutôt que la voiture pour leurs déplacements sont des visiteurs qui, s'ils ne séjournent pas nécessairement plus longtemps que la clientèle traditionnelle, dépensent généralement davantage ${ }^{2}$. Il faudra mieux cibler les efforts de marketing dans les cinq prochaines années - plutồt què de tenter d'intéres-

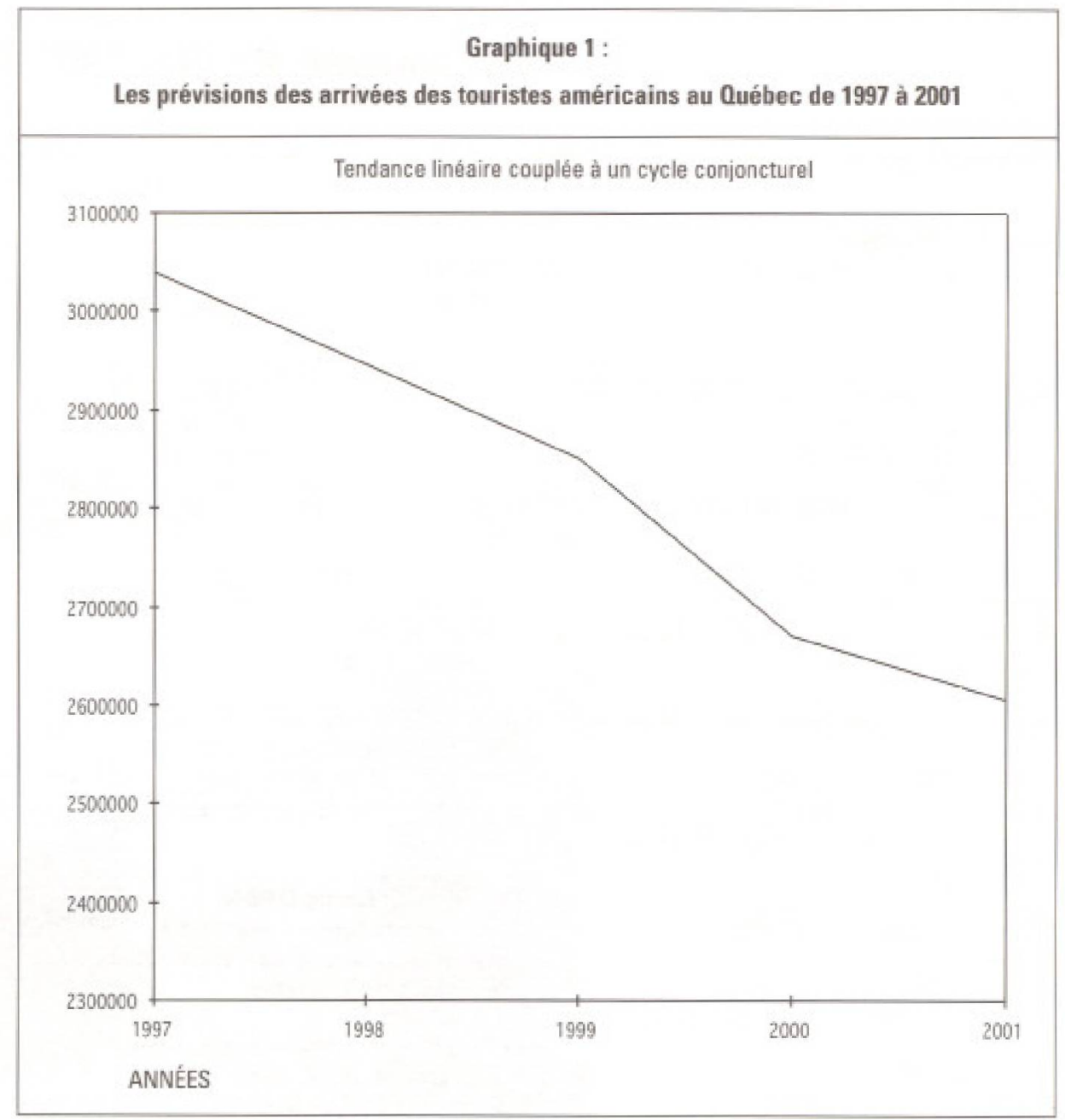

ser a l'Américain moyen * d'une région - de façon à rejoindre les niches de marché les plus lucratives. Faute d'une croissance des visites d'ici l'an 2001, l'avenir est aux longs séjours et à la croissance des dépenses, ce qui, représente tout un défi devant le fractionnement des vacances observé depuis au moins cinq ans dans la plupart des pays industrialisés. À moins que l'avenir du tourisme au Québec ne se trouve ailleurs qu'aux États-Unis...

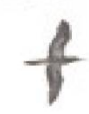

\section{NOTES}

1 Touristes et excursionnistes.

2 A titre d'exemple, le nombre de visitesprovinces pour affaires correspond a seulement $29 \%$ du volume des visites d'agrément, mais les dépenses équivalent à $45 \%$ du total des dépenses d'agrément.

\section{BIBLIOGRAPHIE}

Stafford, Jean, et Daniel Blier (1996), Les clientèles touristiques êtrangères du Québec (19792000) : des mythes à la réalité. Tendances et contre-tendances. Études, matériaux et documents, 10, Département d'études urbaines et touristiques, Université du Québec à Montréal.

The Canadian Tourism Commission (1995), US Leisure Travel Marketing Program. Operational Plan, juin.

The Canadian Tourism Commission (1995), US Leisure Travel Marketing Program. Medium Term Business Plan, avril.

Tourisme Québec (1995), Les touristes anéricains au Quebec 1990 à 1994, Gouvernement du Québec, novembre.

Tourisme Québec (1997), Le tourisme au Québec en 1995. Une réalité économique importanté, Gouvernement du Québec. mars. 